Ann. Zootech., I975, 24 (2), I89-I98.

\title{
INFLUENCE DU RYTHME DE RÉCOLTE SUR LA PRODUCTION DE SPERMATOZÖ̈DES CHEZ LE JEUNE BÉLIER ROMANOV
}

\author{
G. COLAS, D. PERSONNIC, M. COURO'T et R. ORTAVANT \\ avec la collaboration technique de Y. Guéris \\ Station de Physiologie de la Reproduction, \\ Centre de Recherches de Tours, I. N. R. A. \\ B. P. 1, Nouzilly, 37380 Monnaie
}

\section{RÉSUMÉ}

Nous avons étudié l'influence d'une élévation du rythme hebdlomadaire de collecte (du $6^{\mathrm{e}}$ au Io mois : $2 \times$ I éjaculats, du $\mathrm{II}^{\mathrm{e}}$ au I $7^{\mathrm{e}}$ mois : $2 \times 2$ éjaculats, $\mathrm{I} 8^{\mathrm{e}}$ mois : $3 \times 2$ éjaculats) sur les caractéristiques séminales quantitatives de 4 agneaux Romanov dressés au vagin artificiel dès l'âge de 5,5 mois.

Le développement testiculaire (diamètre antéro-postéricur maximum) de ces animaux a été suivi entre le $12^{\mathrm{e}}$ et le $\mathrm{I} 8^{\mathrm{e}}$ mois (février à août).

a) Le volume mensuel moyen de l'éjaculat ( $\mathrm{I}^{\mathrm{er}}$ ou $\mathrm{I}^{\mathrm{er}}+2^{\mathrm{e}}$ éj.) augmente d'une manière régulière, quelle que soit la cadence de récolte $(\mathrm{Y}=0,033 x+0,7 \mathrm{I} 5)$. Sa progression est étroitement liée à celle du développement corporel $(r=0,9 \mathrm{I})$.

b) La concentration et le nombre total de spermatozoides de l'éjaculat augmentent d'une manière significative au cours des 4 semaines qui suivent le changement de fréquence de collecte, lorsque celle-ci passe de 2 à 4 . Lorsque cette fréquence atteint 6 éjaculats par semaine, les deux caractéristiques ne sont pas modifiées de manière significative.

La quantité hebdomadaire de spermatozö̈des éjaculés est multipliée par 2,39 et I,52 lorsque les fréquences de collecte passent respectivement de 2 à 4 et de 4 à 6 . En prélevant les animaux six fois par semaine, on parvient à recueillir $30,28 \times 10^{9}$ spermatozoïdes.

c) Le diamètre antéro-postérieur des testicules diminue de février à juin $(6,47 \mathrm{~cm}$ à $5,90 \mathrm{~cm}$, $\mathrm{P}>0,05)$ et augmente ensuite.

L'accroissement du rythme de récolte entraîne donc chez le Romanov une élévation plus que proportionnelle de la quantité de gamètes éjaculés. Cette production est déjà soumise, chez l'Antenais, aux variations de la photopériode.

\section{INTRODUCTION}

Peu de travaux ont été réalisés sur la production de sperme chez le jeune bélier entre la puberté et l'état adulte. La plupart des auteurs arrêtent en effet leur étude peu après l'apparition des premiers spermatozoïdes dans l'éjaculat (DUN, I955; 
Skinner et Rowson, I968 ; Dyrmundsson et LEES, r972). Seuls Louw et JouberT (I964), ZrNszNer (I97I) ont poursuivi leur analyse au-delà de la pubarté mais le rythme de récolte qu'ils utilisent (I ou 2 éjaculats par semaine) ne permet pas d'avoir une idée précise des possibilités de production de l'animal. On ne sait donc pas à quelle fréquence de collecte on peut soumettre les jeunes mâles et si une modification de celle-ci peut faire varier la quantité de spermatozoïdes éjaculés.

Les coefficients de corrélation calculés entre le poids testiculaire et le développement corporel (COUROT, I962 ; DYRMUNDSSON et LEES, I972) indiquent que la saison ne doit pas avoir beaucoup d'effet sur la croissance des gonades pendant la période prépubère. Or, on sait que chez l'adulte, la saison a une incidence très marquée sur les paramètres testiculaires (ORTAVANT, I958). On peut donc se demander si cet effet n'apparaît pas déjà au début de la phase post-pubère. C'est pourquoi l'influence de la fréquence de collecte et celle de la saison ont été analysées dans cette étude. Nous avons choisi la race Romanov dont on connaît peu de choses chez le mâle si ce n'est sa précocité sexuelle (DESVIGNES et LEFEVRE, I969).

\section{MATÉRIEI, ET MÉ'THODES}

\section{1. -... Animaux expérimentaux}

Quatre agneaux demi-frères nés cntre le 8 et le I0-2-I972 ont été entraînés à la collecte au vagin artificiel dès l'âge de 5,5 mois et récoltés régulièrement pendant un an à partir de l'âge de 6 mois (8-8-1972 au 3I-8-I973).

$$
\text { 2. - Rythme de récolte }
$$

Les béliers étaient sollicités en présence d'une brebis boute-en-train non en chaleur, à des rythmes différents selon leur âge :

- $\mathrm{I}^{\mathrm{re}}$ période : du $6^{\mathrm{e}}$ au $\mathrm{Io}^{\mathrm{e}}$ mois inclus $: 2 \times \mathrm{I}$ éjaculats par semaine (r éjaculat/séance de collecte) ;

- $2^{\mathrm{e}}$ période $: \mathrm{du} \mathrm{II}^{\mathrm{e}}$ au $\mathrm{I} 7^{\mathrm{e}}$ mois inclus : $2 \times 2$ éjaculats par semaine ( 2 éjaculats/séance de collecte) ;

$-3^{\mathrm{e}}$ période : pendant le $\mathrm{I} 8^{\mathrm{e}}$ mois $: 2 \times 3$ éjaculats par semaine ( 3 éjaculats/séance de collecte).

Les collectes étaient toujours précédées d'une fausse monte (Petrov, I969). Les $2^{\mathrm{e}}$ et $3^{\mathrm{e}}$ éjaculats d'une même séance de récolte étaient prélevés à 20 minutes d'intervalle environ.

\section{3. - Examen du sperme}

Ces éjaculats ont été contrôlés pour les caractères suivants :

- motilité initiale (notée de o à 5 après examen au microscope $\times 80$ ) ;

- volume (à o, I ml près);

- concentration (mesurée par néphélométrie);

- nombre total de spermatozoïdes éjaculés.

\section{4. - Mensurations testiculaires}

Le diamètre antéro-postérieur des deux testicules et du scrotum a été mesuré toutes les deux semaines environ à l'aide d'un pied à coulisse. Pour des raisons d'ordre matériel, ces mesures n'ont pu débuter qu'au mois de février alors que les agneaux étaient âgés d'un an. Elles ont été arrêtées au mois d'août et ont toujours été effectuées par la même personne. 


\section{5. - Expression et analyse des résultats}

Les moyennes, calculées avec leur écart-type, sont comparées par analyse de variance (seuil de signification 5 p. Ioo).

Les corrélations ont été calculées à partir des moyennes mensuelles.

L'homogénéité des caractéristiques séminales enregistrées au cours des deux premières périodes est exprimée par les moyennes $\pm s m$ des valeurs absolues des différences entre moyennes hebdomadaires successives.

Les pentes des droites de régression sont comparées suivant la méthode de DaGNeLIE (1970).

\section{RÉSULTATS}

\section{I. - Evolution de la production de sperme (fig. I)}

Les premiers éjaculats ont été obtenus dès l'âge de 6 mois chez les 4 animaux. Nous avons ensuite pu collecter régulièrement ces animaux pendant un an sans rencontrer de difficulté particulière et cela quelle que soit la saison.
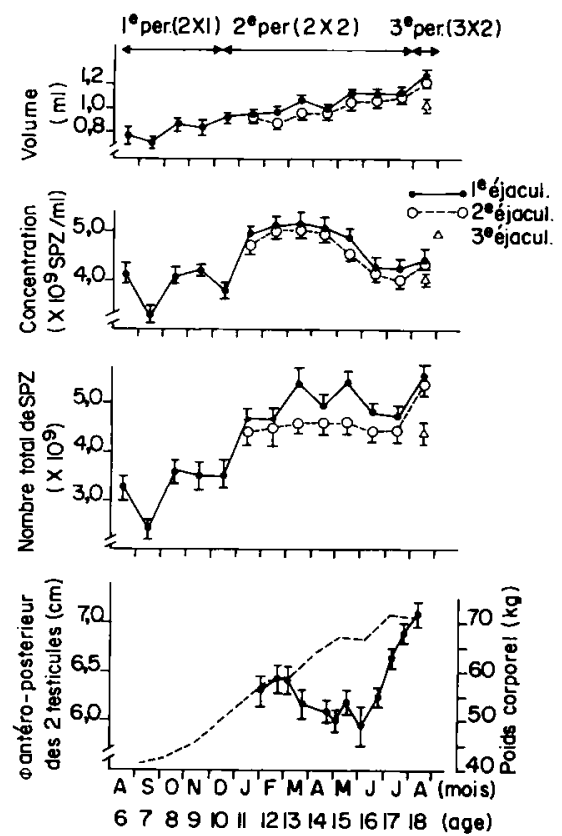

FIG. I. - Evolution des caractéristiques séminales $( \pm s m)$, des dimensions testiculaires (•- $\bullet$ et du poids corporel (-...-) chez le Bêlier Romanov entre 6 et 18 mois

a) Volume.

Ite volume mensuel moyen de l'éjaculat ( $\mathrm{I}^{\mathrm{er}}$ éjaculat pour la $\mathrm{I}^{\mathrm{re}}$ période, $\mathrm{I}^{\mathrm{er}}$ et $2^{\mathrm{e}}$ éjaculats pour la seconde période) croît régulièrement pendant la période expérimentale :

$$
\mathrm{Y}=0,033 x+0,7 \mathrm{I}_{5}
$$


Il est étroitement relié au développement corporel de l'animal :

$$
\mathrm{Y}=0,0 \text { II } x+0,299 \quad(r=0,9 \mathrm{I} ; \mathrm{P}<0,05)
$$

La comparaison des pentes des deux droites de régression (calculées d'après les moyennes mensuelles (fig. I)) correspondant aux périodes I et 2 indique que cette évolution est indépendante des changements de rythmes de récolte :

- Période I : $Y_{1}=0,039 x+0,70 \mathrm{I}$

$$
\mathrm{Y}_{2}=0,036 x+0,682 \quad(\mathrm{P}<0,05) \text {. }
$$

On voit également que les $\mathrm{I}^{\mathrm{er}}$ et $2^{\mathrm{e}}$ éjaculats subissent une évolution parallèle. b) Concentration et nombre total de spermatozoïdes.

Comme on peut le constater sur la figure I, la concentration et le nombre de spermatozoïdes par éjaculat évoluent peu au cours de la $I^{\text {re }}$ période. En effet, lorsque les animaux ont 6 ou ro mois, les valeurs de ces deux caractéristiques sont très voisines (tabl. I).

\section{TABLEAU I}

Concentration et nombre de spermatozoïdes ( $\pm s m$ ) des éjaculats prélevés (V. A.) chez l'Agneau Romanov à 6 et 10 mois $(n=4)$

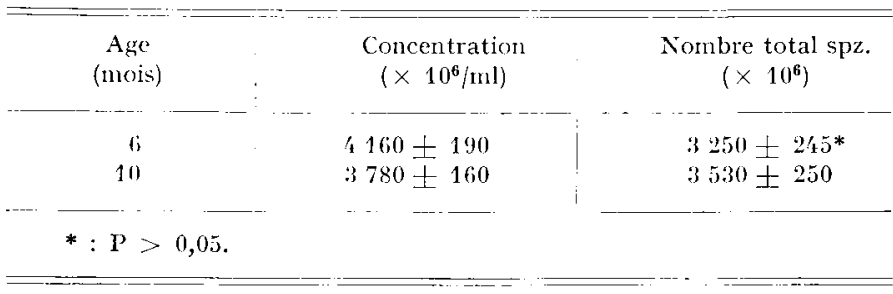

Par contre, elles augmentent brusquement dès le début de la seconde période et conservent des valeurs assez semblables jusqu'au mois d'avril pour la concentration et jusqu'au mois de mai pour le nombre total de spermatozoïdes (fig. I). La comparaison des valeurs moyennes enregistrées au cours de la $\mathrm{I}^{\mathrm{re}}$ ou de la seconde période (arrêtée au 3I-4-I973, deux éjaculats réunis) témoigne de l'importance de ces variations (tabl. 2).

\section{TABLEAU 2}

Evolution des caractéristiques séminales ( $5 \mathrm{sm}$ ) au cours des deux périodes de récolte chez le jeune Romanov $(n=4)$

\begin{tabular}{c|c|c}
\hline P'ériode & $\begin{array}{c}\text { Concentration } \\
\left(\times 10^{6} / \mathrm{ml}\right)\end{array}$ & $\begin{array}{c}\text { Nbre total } \\
\text { spz./éjaculat }\left(\times 10^{6}\right)\end{array}$ \\
\hline $1^{\text {re }(8-8-72 \text { au 5-1-73) }}$ & $\begin{array}{c}3990 \pm 100 \\
5020 \pm 70\end{array}$ & $3270 \pm 110$ \\
\hline Analyse statistique & $P<0,001$ & $P<0,001$ \\
\hline
\end{tabular}


I a concentration diminue de mai à juillet et augmente ensuite au cours du mois d'août ( $3^{\mathbf{e}}$ période). Le nombre total de spermatozoïdes accuse une baisse plus légère en juin et juillet et s'accroît de nouveau au mois d'août.

\section{2. - Influence du rythme de récolte}

a) Lorsque l'on passe de 2 à 4 éjaculats par semaine, la concentration et le nombre total de spermatozoïdes par éjaculat augmentent d'une manière significative au cours des 4 semaines qui suivent le changement de fréquence de collecte. Par contre, le volume de l'éjaculat reste constant (tabl. 3).

b) Lorsque cette fréquence atteint 6 éjaculats par semaine, les caractéristiques séminales ne sont pas modifiées de manière significative (tabl. 3).

\section{TABLEAU 3}

Infuence du changement de rythme de récolte sur les caractéristiques séminales du jeune bélier Romanov $(n=4)$

\begin{tabular}{|c|c|c|c|c|}
\hline Fréquence de collecte & $2 \times 1$ & $2 \times 2$ & $2 \times 2$ & $2 \times 3$ \\
\hline $\begin{array}{l}\text { Période de } \\
\text { comparaison }\end{array}$ & $\begin{array}{c}12-12 \text { au } 5-1-73 \\
n=32\end{array}$ & $\begin{array}{c}6-1-73 \text { au } 2-2-73 \\
n=64\end{array}$ & $\begin{array}{c}10-7-73 \text { au } 3-8-73 \\
n=64\end{array}$ & $\begin{array}{c}7-8-73 \text { au } 31-8-73 \\
n=96\end{array}$ \\
\hline $\begin{array}{l}\text { Volume } \\
\text { (ml) }\end{array}$ & $0,88 \pm 0,0^{\prime}$ & $0,89 \underset{\mathrm{NS}}{ \pm} 0,03$ & $1,1^{\prime} \pm \pm 0,0^{\prime}$ & $1,16 \underset{\mathrm{NS}}{ \pm} 0,02$ \\
\hline $\begin{array}{l}\text { Concentration } \\
\left(\times 10^{6}\right)\end{array}$ & $3950 \pm 200$ & $\begin{array}{c}4860 \pm 110 \\
P<0,001\end{array}$ & $4340 \pm 115$ & $4_{\frac{1}{\mathrm{NS}}} 300$ \\
\hline $\begin{array}{l}\text { Nombre total } \\
\qquad\left(\times 10^{6}\right)\end{array}$ & $3630 \pm=90$ & $\begin{array}{c}4340 \pm 200 \\
P<0,01\end{array}$ & $4990 \pm 190$ & $5050 \underset{\mathrm{NS}}{ \pm} 180$ \\
\hline $\begin{array}{l}\text { Production } \\
\text { hebdomadaire } \\
\left(\times 10^{6}\right)\end{array}$ & 7270 & 17370 & 19980 & $30 \stackrel{-80}{0}$ \\
\hline $\begin{array}{l}\text { Augmentation } \\
\text { relative }\end{array}$ & & 39) & & $1,52)$ \\
\hline
\end{tabular}

Le nombre de spermatozoïdes recueillis chaque semaine est multiplié par 2,39 lorsqu'on double la fréquence de collecte $\left(7270 \times \operatorname{IO}^{6}\right.$ vs $17370 \times 10^{6} \mathrm{spz}$ respectivement pour 2 et 4 éjaculats par semaine) et par I,52 lorsque celle-ci passe de 4 à 6 (I9 $980 \times 10^{6}$ vs $30280 \times 10^{6} \mathrm{spz}$, tabl. 3$)$.

La variabilité des moyennes hebdomadaires des caractéristiques séminales du I $^{\text {er }}$ éjaculat est toujours plus élevée au cours de la $I^{\text {re }}$ ( 2 éjaculats/semaine) que de la seconde période (4 éjaculats/semaine) (tabl. 4). Les différences ne sont cependant significatives que pour la concentration. 
TABLEAU 4

Influence du rythme hebdomadaire de collecte sur l'homogénéité des caractéristiques séminales

(Cette homogénéité est exprimée par la moyenne $+s m$ des valeurs absolues des différences entre moyennes hebdomadaires successives)

\begin{tabular}{|c|c|c|}
\hline & \multicolumn{2}{|c|}{ Éjaculats/semaine } \\
\hline & $2 \times 1$ & $\begin{array}{c}2 \times 2 \\
\text { (1 er éjaculat) }\end{array}$ \\
\hline Volume (ml) & $0,128 \pm 0,021$ & $0,085 \pm 0,015$ \\
\hline Concentration $\left(\times 10^{6} / \mathrm{ml}\right)$ & $397 \pm 56$ & $272 \pm 36^{*}$ \\
\hline $\begin{array}{l}\text { Nombre total de } \\
\text { spermatozoìdes }\left(\times 10^{6}\right)\end{array}$ & $562 \pm 118$ & $445 \pm 67$ \\
\hline$* \mathrm{P}<0,05$ & & \\
\hline
\end{tabular}

\section{3. - Evolution de la croissance testiculaire}

Contrairement au poids corporel qui connaît une croissance régulière jusqu'au $\mathrm{I} 7^{\circ}$ mois $(\mathrm{Y}=3$, Oro $x+38,042)$, le diamètre antéro-postérieur moyen des deux testicules diminue de $6,47 \pm 0, \mathrm{I} 4 \mathrm{~cm}$ à $5,93 \pm 0, \mathrm{I} 9 \mathrm{~cm}$ du I9 février au 4 juin I973, puis augmente rapidement pour atteindre $7, \mathrm{II} \pm 0, \mathrm{I}_{5} \mathrm{~cm}$ le 7 aôtt I973. Les différences enregistrées ne sont pas significatives mais elles sont présentes et de même sens chez les quatre animaux.

\section{DISCUSSION}

Cette étude montre que les caractéristiques séminales du jeune bélier Romanov entre 6 et 18 mois évoluent sous l'influence de facteurs différents.

\section{I. - Volume}

Le volume du sperme éjaculé augmente régulièrement avec l'âge de l'animal. Cette constatation est en accord avec celles de Louw et JouberT (I964), SKINNER et Rowson (I968) bien que les conditions expérimentales soient différentes notamment en ce qui concerne la technique de prélèvement (électroéjaculation). Elle est d'ailleurs valable pour la plupart des espèces domestiques. Par contre, il n'a jamais été démontré, comme nous l'avons fait dans notre étude, que cette évolution est indépendante du rythme hebdomadaire de collecte au moins dans le cas où celui-ci ne dépasse pas 6 éjaculats. Le volume de semence recueillie n'est donc fonction chez le jeune bélier que de sa croissance corporelle, comme en témoigne la corrélation 
calculée entre ces deux paramètres. On voit d'ailleurs sur la figure I qu'ils sont les seuls à augnenter d'une façon régulière.

L'examen de la droite de régression (volume, âge) établie pour l'ensemble de 1a période expérimentale $(y=0,033 x+0,7$ I5) laisse penser que le volume moyen de l'éjaculat, déjà très élevé ( $\mathrm{I}, \mathrm{I} 6 \mathrm{ml}$, tabl. 3), va encore augmenter. Or ce volume est déjà plus grand que celui qui a été obtenu à la même saison chez de jeunes sujets entraînés seulement à l'âge de I7 mois ou d'animaux adultes collectés de façon sporadique ( ${ }^{1}$ ). Il semble donc qu'un dressage précoce et des récoltes régulières permettent d'augmenter la quantité de sperme éjaculé chez le bélier.

Ces résultats montrent aussi que la croissance du volume de l'éjaculat n'est pas affectée par les fluctuations saisonnières de la photopériode au moins pendant les dix-huit premiers mois de la vie de l'animal.

\section{2. - Concentration et nombre de spermatozoides}

A la différence du volume, la concentration et la quantité de spermatozoïdes des éjaculats sont liées étroitement à la fréquence des collectes. Ceci ne peut être mis en évidence d'une manière précise qu'à la condition de maintenir un rythme de prélèvement assez élevé. Le rythme adopté au cours de la première période était sans doute insuffisant. C'est pourquoi le nombre de cellules sexuelles recueillies n'a pas augmenté d'août à novembre I 972 bien que la photopériode ait été favorable à la spermatogenèse (ORTAVANT, I958; PELLETIER, I97I) et la croissance corporelle régulière.

La brusque élévation de la concentration du sperme dès le début de la seconde période, c'est-à-dire au moment où la cadence de récolte passe de 2 à 4 éjaculats par semaine est difficile à expliquer. En général on observe une relation inverse : plus un animal est sollicité, plus la concentration de ses éjactulats diminue. Dans notre expérience, ce phénomène pourrait provenir d'une augmentation de la production spermatogénétique ou bien d'une amélioration des contractions des canaux épididymaires et des canaux déférents. La première hypothèse doit être écartée puisque l'effet observé est quasi immédiat comme l'indique le tableau 3. La seconde par contre est plus vraisemblable. On sait en effet qu'au moment de l'émission du sperme, i1 y a de violentes contractions des parois musculaires du canal déférent (EsSENHIGH et al., r969). Il est donc possible qu'en augmentant le nombre de collectes sur un jeune animal on stimule la qualité et l'efficacité de ses réflexes d'éjaculation.

L'accroissement de la fréquence des récoltes est également très favorable à l'obtention d'un grand nombre de spermatozoïdes. Ceci ne peut résulter que d'une amélioration des conditions de récupération de ces gamètes. On sait en effet d'après les travaux de LiNo et BRADEN (I972), TISChNer (I972) que chez le bélier non soumis à la collecte, le sperme s'élimine d'une façon permanente par l'urine. Ces pertes " non contrôlées " sont d'autant plus faibles que le rythme de prélèvement est élevé. Autrement dit, chez l'Agneau, une basse fréquence de collecte ne permet de récupérer qu'une faible proportion de la production spermatique.

(1) Sur 4 demi-frères Romanov de même âge, entraînés à la collecte, dix-sept mois seulemant après la naissance, nous avons obtenu à la même époque en moyenne $\mathrm{I}, \mathrm{or} \pm 0,04 \mathrm{ml}$ contre $\mathrm{I}, \mathrm{r} 9 \pm 0,03 \mathrm{ml}$ ( 48 fjaculats par lot pendant $I_{5}$ jours, $\mathrm{P}<\mathrm{o}$,oor). Par ailleurs, sur des sujets adultes de même race, collectés de manière sporadique, nous avons enregistré un volume de $0,97 \pm 0,04 \mathrm{ml}(\mathrm{P}<0,05)$ sur un total de 40 éjaculats. 
Nous avons recueilli en moyenne $30,2 \times 10^{9}$ spermatozoïdes par semaine sur des animaux encore en cours de croissance et soumis à un régime hebdomadaire de 6 éjaculats. Le tableau I montre que les caractéristiques séminales n'ont pas diminué

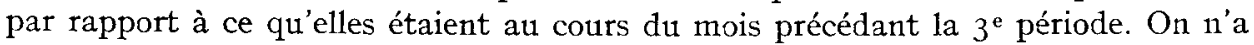
donc pas atteint chez ces béliers les possibilités de production maximum. Si l'on se réfère aux travaux d'ORTAVANT (I958), Lino et BRADEN (I972) sur des sujets adultes, on voit que la proportion de spermatozoïdes récupérés par rapport à la production totale est supérieure à 70 . Ces chiffres se situent entre ceux obtenus chez le Taureau, dont le rendement ne dépasse guère 50 p. Ioo (AMANN et Almouist, I962 ; Amann, 1970) et le verrat chez lequel une seule collecte par semaine suffit à recueillir la presque totalité des gamètes produits (StgNoret, du MESNIL du Buisson et BARITEAU, I97I).

L'examen de la courbe I montre que la croissance testiculaire n'est pas régulière chez l'antenais. Le diamètre antéro-postérieur des testicules diminue légèrement chez les quatre animaux de mars à mai pour augmenter à nouveau au mois de juin et dépasser en fin d'expérience le niveau atteint en février. L'accroissement de $\mathrm{r}, 3 \mathrm{~cm}$ en 3 mois représente une augmentation d'environ $20 \mathrm{p}$. Ioo. Celle-ci débute à peu près au moment où la photopériode commence à décroître. La corrélation entre le poids testiculaire et le diamètre antéro-postérieur étant très élevée (COL,YER, I97I), on peut dire que l'incidence de la lumière sur le volume testiculaire se manifeste chez l'antenais comme chez l'adulte, tout au moins en ce qui concerne la durée du phénomène (PELLETIER, I97I). Elle est prépondérante par rapport à celle de la croissance corporelle puisqu'elle entraîne pendant un certain temps une régression de la gonade. Le même phénomène a été observé chez le Cerf (Lincoln, I97I). En conjuguant l'influence du développement corporel et celle d'une photopériode favorable, il doit donc être possible d'amener l'animal à atteindre plus rapidement son état adulte.

La lumière est sans effet sur le volume de sperme produit, ce qui est normal si l'on se réfère à ce que nous avons dit plus haut. En revanche, elle provoque une baisse importante de la concentration dès le mois de mai c'est-à-dire environ deux mois après celle enregistrée pour le testicule. On observe d'ailleurs le même délai lorsque le diamètre antéro-postérieur augmente à nouveau. Or, ce délai correspond à la durée du cycle spermatogénétique (ORTAVANT, I958) ajoutée à celle du transit des spermatozoïdes dans l'épididyme (Amir et OR'TAVANT, I968).

Les variations du nombre total de spermatozoïdes sont moins accusées et de plus courte durée. Ceci peut s'expliquer à la lumière de ce que nous venons de dire au sujet du volume et de la concentration des éjaculats.

En conclusion, l'augmentation du rythme de collecte chez l'Agneau Romanov ne diminue ni la concentration ni le nombre de spermatozoïdes des éjaculats obtenus. Elle entraîne au contraire une élévation plus que proportionnelle de la quantité de gamètes éjaculés et permet de récupérer une grande partie de la production spermatique. Chez l'antenais, cette production reste soumise aux variations de la photopériode, mais d'une manière moins étroite que chez l'adulte (ORTAVANT et Thibaurit I956; SMITH et GoRDON, I967). 


\title{
SUMMARY
}

\author{
EFFECT OF FREQUENCY OF COLLECTION ON PRODUCTION \\ OF EJACUI,ATED SPERMATOZOA IN THE YOUNG ROMANOV RAM
}

We have studied the influence of an increase of the weekly frequency of collection on the concentration, the volume and the total number of spermatozoa of the ejaculates obtained from 4 Romanov ram-lambs. The animals, born between the 8 th and the 1 oth of february 1972 , have been trained to the A. V. from the age of 5,5 months (August) and regularly collected every week at different intensities according to their age :

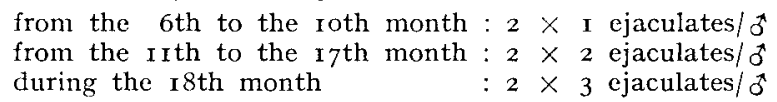

We have also analysed the effect of the season on the testicular development. For this purpose we have measured the maximum antero-posterior diameter of the testicles of the four animals between the age of 12 and 18 months (february to august).

a) The mean monthly volume of the ejaculate (first or first and second ejaculate) increases regularly irrespective of the rhythm of collection ( $\mathrm{Y}=0,033 x+0,7$ I 5 ). Its progression is closely related to that of the physical development $(r=0,9 \mathrm{I})$.

b) The concentration and the total number of spermatozoa increase significantly during the 4 weeks after the changing of frequency of collection, when the latter turns from 2 to 4 . When this frequency reaches 6 ejaculates per week, these two characteristics are not significantly modified.

The mean weekly sperm output is multiplied by 2,39 and 1,52 when the frequencies of collection turn respectively from 2 to 4 and 4 to 6 . With a rhythm of six ejaculates per week, sperm production reaches $30,28 \times \mathrm{I}^{9} \mathrm{spz}$.

c) The antero-posterior diameter declines from february to june $(6.47 \mathrm{~cm}$ to $5.90 \mathrm{~cm}, \mathrm{P}>$ $0,05)$ and increases afterwards.

The acceleration of the intensity of collection so produces in the Romanov ram a more than proportional elevation of the sperm output, when the subjects have been trained to the $A$. V. in their young days. At the age of $\mathbf{I} 2$ to 18 months, their sperm production depends upon the influence of the photoperiodism. However, this influence is less important than that observed in the adult.

\section{RÉFÉRENCES BIBLIOGRAPHIQUES}

Amann R. P., Ig7o. Sperm production rates. In : The Testis. Acad. Press, N. Y. and Lond., 1, 433-482.

Amani R. P., Almguist J. O., r962. Reproductive capacity of dairy bulls. VIII. Direct and indirect measurement of testicular sperm production. J. Dairy Sci, 48, 774.

Amir D., Ortavant R., ig68. Influence de la fréquence des collectes sur la durée de transit des spermatozoïdes dans le canal épididymaire du bélier. Ann. Biol. anim. Bioch. Biophys., 8, I95-207.

Colyer R. J., I97I. Development of the testis and epididymis of the Clun Forest Ram. J. Agric. Sci. $C a m b ., 76,433-44$ I.

Courot M., 1962. Développement du testicule chez l'Agneau. Établissement de la spermatogenèse. Ann. Biol. anim. Bioch. Biophys., 2, 25-4I.

DAGNelie P., x97o. Théorie et méthodes statistiques. II. Les méthodes de l'inférence statistique. Éditions J. Duclot, S. A., Gembloux.

Desvignes A., Lefevre C., I969. Les aptitudes de la race ovine Romanov et ses possibilités d'utilisation pour l'amélioration de la production d'agneaux de boucherie. Assoc. Franc. Zootech., 11, I3.

Desvignes A., r971. La race ovine Romanov. Ann. Zootech., 20, 353-370.

Dun R. B., 1955. Puberty in Merino rams. Aust. Vet. J., 31, I04-106.

Dyrmundson D. R., Lees J. L., r972. Puberal development of Clun Forest ram lambs in relation to time of birth. J. Agric. Sci. Camb., 79, 83-89.

lissenhigh D. M., Ardran G. M., Hovell G. J. R., Sмith J. C., I969. The vesical sphincters and ejaculation in the ram. Brit. J. of Urol., 2, 190-196. 
Hovell G. J. R., Ardran G. M., Essenhigi D. M., Smith J.-C., I969. Radiological observations on electrically induced ejaculation in the ram. J. Reprod. Fert., 20, 383-388.

Lincoln C. A., I97I. Puberty in a seasonally breeding male the red deer stag (Cervus elaphus L.). J. Reprod. Fert., 25, 4I-54.

Lrno B. F., Braden A. W. H., r972. The output of spermatozoa in rams. I. Relationship with testicular output of spermatozoa and the effect of ejaculations. Aust. J. Biol. Sci., 25, 35r-358.

Louw O. F. J., Joubert D. M., 1964. Puberty in the male Dorper sheep and Boer goat. S. Afr. $J$. A gric. Sci., $\mathrm{y}$, 509-520.

Ortavant R,, Thibault C., I956. Influence de la durée d'éclairement sur la production spermatique du bélier. C. R. Séances. Soc. Biol., CL, 2, 358-36r.

Ortavant R., 1958. Le cycle spermatogénétique chez le Bétier. D. Sc., Thèse, Paris, I27 p., C. N. R. S., $n^{\circ} 3$ II8-0-3990.

Pelletier J., I97I. Influence du photopériodisme et des androgènes sur la synthèse et la libération de $L H$ chez le Bélier. D. Sc. Thèse, Paris, 243 p., C. N. R. S., nº A. O. 544 I.

Pficov E., I969. The effect of sexual excitement on spermogram data and on the fertilizing capacity of rams.

Signoret J.-P., du Mesnil du Buisson F., Bariteau F., i97i. L'insémination artificielle porcine. Bull. Tech. Inform. Minist. Agric., 25\%, I57-162.

Skinner J. D., Rowson I. E. A., I968. Puberty in Suffolk and cross-bred rams. J. Reprod. Fert., 16, 479 .

Smith P., Gordon I., I967. Seasonal and breed variations in the semen characteristics of rams in Ireland. Irish Vet. J., 21, 222-233.

Tischner M., I972. The role of the vasa deferentia and the urethra in the transport of semen in rams. Acta A grar. Silvest. Ser. Zootec., XII, 77-1r4.

Zinszner F, I97I. Étude quantitative et qualitative de la production de sperme chez l'Agneau. Menoire de fin d'études, E. N.S.F. A., Rennes. 